\title{
Guidelines on oral anticoagulation: second edition
}

\author{
British Society for Haematology \\ British Committee for Standards in Haematology \\ Haemostasis and Thrombosis Task Force
}

\section{Introduction}

The guidelines document on oral anticoagulation first appeared in 1984. Since then the international system for the standardisation of the prothrombin time in anticoagulant control, based on International Normalised Ratios (INR), has become widely accepted. ${ }^{1-3}$ The introduction of INR, combined with the change in the United Kingdom from human brain to rabbit thromboplastin in 1986, has created a need for this second edition.

A survey on oral anticoagulation conducted for the British Society for Haematology (BSH) via the United Kingdom National External Quality Assessment Scheme (NEQAS) in blood coagulation in 1982 estimated that 2.3 million prothrombin time tests were performed annually in the United Kingdom for purposes of oral anticoagulant control and that about a quarter of a million people were receiving this treatment each year. The numbers are now much greater.

\section{Properties of oral anticoagulants}

The commonly used oral anticoagulant drugs are those derived from 4-hydroxycoumarin, of which the most widely used is warfarin, named after the Wisconsin Alumni Research Foundation. Other drugs listed in the British National Formulary are nicoumalone and phenindione. Phenindione is now rarely used due to hypersensitivity reactions.

Oral anticoagulants antagonise vitamin $K$, required for the gamma carboxylation of certain glutamic acid residues which facilitate calcium binding of coagulation factors II, VII, IX, $\mathrm{X}$ and the anticoagulant factors protein $\mathrm{C}$ and protein S. Acarboxy forms which circulate in patients treated with coumarin drugs are devoid of procoagulant properties. The rate of disappearance from the circulation of these vitamin $\mathrm{K}$ dependent clotting factors depends on their respective half lives, which range from six to 60 hours.

\section{Use of oral anticoagulants}

Coumarin drugs are used for the prevention and control of thrombo-embolism. The following are the conditions for which oral anticoagulants are used, but there are wide differences of opinion on their indications.
Short term to medium term (up to 12 months)

Prophylaxis of deep vein thrombosis including high risk surgery; ${ }^{4-6}$ myocardial infarction, anterior myocardial infarction, three months minimum ${ }^{7}$; established deep vein thrombosis, three months minimum ${ }^{8}$; xenograft heart valve replacements, three months minimum ${ }^{9}$; pulmonary embolism, three to six months ${ }^{8}$; coronary artery bypass graft, up to two months. ${ }^{10}$

\section{Long term}

Recurrent venous thrombo-embolism ${ }^{8}$; embolic complications of rheumatic heart disease and atrial fibrillation ${ }^{11}$; cardiac prosthetic valve replacement and arterial grafts. $^{912}$

POSSIBLE INDICATIONS FOR LONG TERM TREATMENT

Congenital antithrombin III deficiency with clinical thrombosis; congenital protein $\mathrm{C}$ or $\mathrm{S}$ deficiencies with clinical thrombosis; transient ischaemic cerebral artery syndrome including basilar and vertebral artery syndromes; lupuslike anticoagulant with clinical thrombosis.

\section{Pregnancy}

The decision on whether to use oral anticoagulant drugs during pregnancy is still a matter of clinical judgment because of the balance of risk between mother and fetus.

The main disadvantage of vitamin $K$ antagonists during pregnancy is that they cross the placenta. Warfarin may be teratogenic during the first trimester, resulting in warfarin embryopathy, and is associated with an increasing risk of fetal haemorrhage as pregnancy progresses. The risk is maximal during labour and delivery. A warning on the dangers of becoming pregnant while taking oral anticoagulants is given in the national anticoagulant treatment booklet.

When anticoagulation during pregnancy is deemed essential, intravenous or self-administered subcutaneous heparin in adjusted doses should be considered. (For further details of 
heparin administration see $\mathrm{BSH}$ guidelines on heparin). Long term heparin administration, however, carries a risk of maternal osteoporosis. Women who have prosthetic heart valves or who have mitral valve disease or atrial fibrillation, or both, may be more safely maintained on warfarin. Wherever possible, oral anticoagulants should be avoided during the first trimester. In any patient given warfarin during the second and third trimesters frequent monitoring and dose review are essential. For all patients warfarin must be replaced by heparin at around 36 weeks.

If heparin is continued during labour its dose should be carefully monitored. After delivery full dose heparin may usually be reintroduced 12-24 hours after completion of the third stage. Oral anticoagulants may be given 24-48 hours after delivery. Introduction of vitamin $\mathrm{K}$ antagonists should be progressive, with no initial loading dose. Heparin should be continued until the INR is in the desired therapeutic range. Anticoagulated mothers of healthy infants should not be discouraged from breast feeding.

\section{Contraindications}

These are seldom absolute and depend on individual patients (box).

\begin{tabular}{|c|c|}
\hline General: & $\begin{array}{l}\text { Mental impairment } \\
\text { Uncooperative } \\
\text { patients } \\
\text { Alcoholism }\end{array}$ \\
\hline Cardiovascular: & Hypertension \\
\hline Renal: & $\begin{array}{l}\text { Sustained increase of } \\
\text { blood urea above } \\
10 \mu \mathrm{mol} / \text { litre }\end{array}$ \\
\hline Neurological: & $\begin{array}{l}\text { Recent non-embolic } \\
\text { cerebrovascular } \\
\text { accidents, recent } \\
\text { surgery, or trauma } \\
\text { to central nervous } \\
\text { system and eye }\end{array}$ \\
\hline Gastrointestinal: & $\begin{array}{l}\text { Inflammatory bowel } \\
\text { disease, peptic } \\
\text { ulcer, oesophageal } \\
\text { varices }\end{array}$ \\
\hline Liver disease: & $\begin{array}{l}\text { Uncompensated } \\
\text { cirrhosis }\end{array}$ \\
\hline Haematological: & $\begin{array}{l}\text { Pre-existing } \\
\text { haemostatic defect }\end{array}$ \\
\hline
\end{tabular}

\section{Dose and control of anticoagulant} treatment

The dose of anticoagulant drug depends on biological assay of the induced coagulation defect, measured by the prothrombin time, which is the recalcification time of plasma after the addition of a tissue thromboplastin. Thromboplastin reagents derive from a variety of sources and usually give different prothrombin times for the same test plasma because of differing potencies and responsiveness to depression of the vitamin $\mathrm{K}$ dependent clotting factors. All thromboplastin reagents should be labelled with an International Sensitivity
Index (ISI) which quantifies their responsiveness to the effects of anticoagulants in terms of the WHO Primary Reference Preparation which has an assigned value of $1 \cdot 0$. This permits reporting as INR. INR are virtually identical with the formerly recommended British Ratios (BR). ${ }^{3}$

After clinical appraisal a baseline prothrombin time should be determined whenever possible to assess liver function before the initial dose of warfarin is administered. The usual adult induction dose of warfarin is $10 \mathrm{mg}$ on the first day and $10 \mathrm{mg}$ on the second day (a larger loading dose is not now recommended).

The dose schedule should be reduced in the following conditions: prolonged baseline prothrombin time; abnormal liver function tests; congestive cardiac failure; parenteral feeding; less than average body weight; age of over 80 years.

The daily dose should be given, if possible, at a fixed time-for example, 1800 hours. The INR must be determined on the day of treatment and the dose of warfarin adjusted accordingly. The maintenance dose is usually between 3 and $9 \mathrm{mg}$ warfarin, although the pharmacological response varies widely among patients and even in the same patient over a period of treatment. If the patient is receiving concurrent heparin treatment by continuous infusion and is being maintained by the activated partial thromboplastin time (APTT) at less than two and a half times control reading (see BSH guidelines on heparin treatment), oral anticoagulant dose can still be based on the prothrombin time from a specimen collected without discontinuing heparin infusion. The prothrombin time prolongation by heparin at therapeutic concentrations is slight. If the dose of heparin is excessive it may be necessary to withhold treatment for three to four hours and repeat the test. Repeat prothrombin times are normally required daily or on alternate days in the early days of treatment, or at longer intervals later, depending on the response. If a patient is well stabilised the interval of prothrombin time testing can be gradually extended to eight weeks as an outpatient. Where there is a pronounced change in the clinical state or in concomitant drug treatment, especially if the drug is known to interact with warfarin, the $N$ prothrombin time must be checked more frequently. When a known potentiating or antagonising drug is administered or with- $O$ drawn, the dose of warfarin should either be $\mathbb{\Phi}$ adjusted or the prothrombin time checked more frequently. Other drugs which are recog- $\frac{0}{0}$ nised as possibly synergistic with oral anticoagulants can be given in normal doses but $\frac{\rho}{\mathbb{Q}}$ with more frequent monitoring.

\section{Therapeutic ranges}

As experience of the improved anticoagulant control in the United Kingdom afforded by the? provision of standardised low ISI (high sensitivity) thromboplastin and external quality assessment has increased, more specific and, in some instances, more intense therapeutic ranges have been used with confidence. The BSH 
Table 1 Suggested INR ranges in various conditions

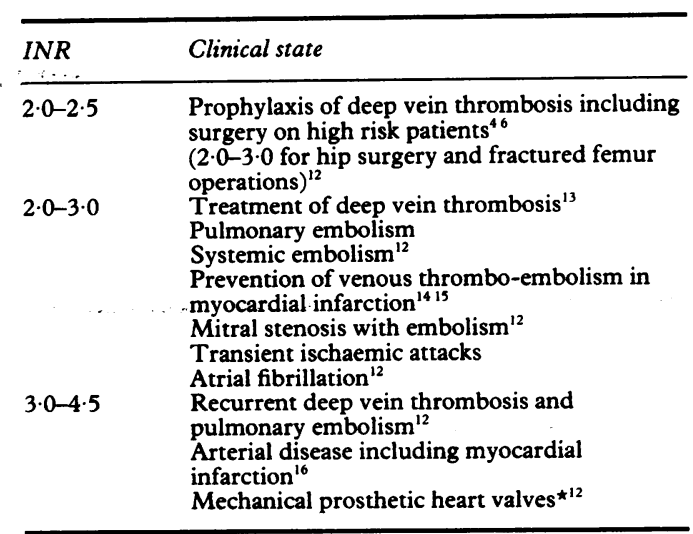

^A recent randomised study in patients with tissue prosthetic heart valves indicated that control at a less intensive INR range of 2.0-3.0 was effective and safe."

proposals (table 1) were based on the results of a NEQAS survey on current practice, supported in respect of prevention and treatment of deep vein thrombosis by previous randomised clinical trials. ${ }^{4-6}$

Although surgical procedures, including dental extractions, may be associated with an increased risk of bleeding if these are performed under anticoagulant cover, two randomised studies ${ }^{56}$ have shown that the bleeding risk is low if the INR on the day of operation is between 2.0 and 2.5 with a low ISI thromboplastin. In practice an INR of not greater than 2.0 is recommended at the time of surgery but the risk of bleeding depends on the clinical circumstances.

\section{Side effects}

Apart from haemorrhage, side effects encountered with oral anticoagulants have been mostly with the indanediones, principally phenindione, the use of which has been largely discontinued as sensitivity reactions were relatively common. Skin rashes and alopecia have been reported as rare complications with coumarin drugs. Cutaneous necrosis associated in some cases with heterozygous protein $\mathrm{C}$ or protein $\mathrm{S}$ deficiency is due to capillary thrombosis during the induction phase. In such patients administration of heparin during the first few days of treatment with slow induction of warfarin is advised. Poor control may give similar problems at a later stage in these patients. Demineralisation of bone has been reported with prolonged oral anticoagulant treatment in young children. Acute intestinal obstruction is a rare complication of mesenteric haemorrhage.

\section{Causes of prolonged prothrombin time} and haemorrhage

These are due to relative overdose or increased sensitivity by the patient either as a consequence of a change in patient's physical state-any severe illness, interaction with a potentiating drug, and withdrawal of antagonistic drugs.

The following pathological disorders have a confirmed potentiating effect: alcohol excess, either acute or chronic; cardiac failure; cholestasis; diarrrhoea (enteritis); fever; gastrocolic fistula; hypoalbuminaemia; liver damage (including exposure to organic solvents such as petrol and possibly halothane in general anaesthesia); malnutrition; severe weight reduction regimens; renal impairment; thyrotoxicosis.

Drugs used for the following have a high potentiating effect:

Gastrointestinal tract:

Cimetidine.

Cardiovascular system:

Amiodarone, clofibrate, bezafibrate, dextrothyroxine, quinidine, sulphinpyrazone.

Infections:

Co-trimoxazole, metronidazole.

Endocrine system:

Anabolic steroids, danazol, glucagon, thyroxine.

Musculoskeletal and joint disease:

Aspirin and the salicylates, azapropazone, feprazone, sulphinpyrazone.

Malignant disease:

Tamoxifen.

Alcoholism:

Disulfiram.

A fuller list of drugs available in the United Kingdom which may potentiate or antagonise anticoagulants is given in the Appendix.

\section{Recommendations for concomitant drug treatment}

Analgesics used for headaches and minor trauma containing dextropropoxyphene hydrochloride or paracetamol, or a combination with co-proxamol, (Distalgesic), are relatively safe for occasional self-medication in doses of two to four tablets a day, once or twice a week, but regular ingestion of six to eight tablets daily for chronic pain may require reduction of oral anticoagulant dose.

While most interacting drugs operate by potentiating or antagonising the action of coumarin-like drugs, others, such as aspirin, sulphinpyrazone, and non-steroidal antiinflammatory drugs, also act by impairing platelet function and so enhance the risk of bleeding, especially in the gastrointestinal tract. Aspirin also has an undesirable localised irritant effect on the gastric mucosa, resulting in an increased incidence of haematemesis. Thus prothrombin time control alone will not reflect the potential risk of bleeding.

\section{Reversal of oral anticoagulant treatment} Recommendations on reversal of oral anticoagulant treatment have to be influenced by the risks of inducing hepatitis and human immunodeficiency virus (HIV) infection with plasma concentrates and the possible thrombotic dangers of over correction by vitamin $\mathrm{K}$, especially in patients with prosthetic or replacement heart valves (table 2 ). With these risks in mind, the following recommendations are made with the warning that four to six hours must be allowed for an adequate clinical response to vitamin $K_{1}$ in the average patient. 
Table 2 Recommendations on reversal of oral anticoagulant treatment

A Life threatening haemorrhage Immediately give $5 \mathrm{mg}$ vitamin $\mathrm{K}_{1}$ by slow intravenous infusion and a concentrate of factor II, IX, X, with factor VII concentrate (if available)

The dose of concentrate should be calculated based on 50 iu factor IX/kg body weigh

If no concentrate is available fresh frozen plasma should be infused (about 1 litre for an adult) but this may not be be infused (abc

B Less severe haemorrhage such as haematuria and epistaxis Withhold warfarin for one or more days and consider giving vitamin $K_{1} 0.5-2.0 \mathrm{mg}$ intravenously

C INR of $>4.5$ without haemorrhage Withdraw warfarin for one or two days then review

D Unexpected bleeding at therapeutic levels Investigate possibility of underlying cause such as unsuspected renal or alimentary tract disease

\section{Causes of INR of less than 2.0}

Failure to achieve an adequate degree of anticoagulation may be due to inadequate dose, patient non-compliance, hereditary resistance, acquired resistance to warfarin, improvement in the patient's general condition, dietary changes or antagonistic drug interaction. Plasma warfarin concentration may assist in diagnosis of non-compliance. Barbiturates and preparations containing vitamin $\mathrm{K}$ are particularly prone to cause low INR values; other drugs prescribed in the United Kingdom that are antagonistic to oral anticoagulants are listed in the Appendix. Acquired drug resistance, although a rare occurrence, may be difficult to manage. Changing to an alternative drug may be beneficial, but resistance is often encountered with other oral anticoagulant compounds.

\section{Withdrawal of oral anticoagulant treatment}

Whether treatment should be withdrawn abruptly or gradually withdrawn ("tailed off") is still debatable. Theoretically, the "rebound hypercoagulability" which results from sudden discontinuation might predispose to rebound thrombosis. Some clinicians tail off long term treatment over several weeks but withdraw short term treatment suddenly.

\section{Organisation of oral anticoagulant control service}

Each haematology service or department should provide a clinical and laboratory service for the control of oral anticoagulant treatment. This should be concerned solely with the supervision of anticoagulant doses and related problems. Prescription of oral anticoagulant drugs must be the responsibility of a registered medical practitioner.

\section{INPATIENT SERVICE}

The consultant haematologist should be willing to advise on the hospital's policy for the control of oral anticoagulant drugs. He or she should provide advice on the selection of patients and the appropriate therapeutic range and should counsel patients on oral anticoagulant drugs. A survey on oral anticoagulation conducted for the British Society for Haematology through the United Kingdom National External Quality Assessment Scheme (NEQAS) indicated that half of inpatient anticoagulant control is undertaken by consultant haematologists alone or with junior haematology staff. In some additional instances responsibility is shared with consultant colleagues. Where anticoagulant treatment gets out of control or requires to be reversed, the haematologist should advise on measures to correct this and provide blood products as necessary.

The haematologist should organise a laboratory service for the control of oral anticoagulant drugs according to the principles set out in these guidelines. There should be formal referral in writing of patients to the anticoagulant clinic with treatment authorisation signed by the requesting medical practitioner. For inpatient doses, this will normally be based on the prothrombin time of venous blood samples sent to the laboratory. Written reports giving INR values should be issued and the results entered in the ward, into an anticoagulant control chart.

\section{OUTPATIENT SERVICE}

Patients on discharge from hospital should normally be referred to a consultant haematologist for the control of outpatient treatment. Each haematology department or service should be prepared to organise outpatient clinics for the control of oral anticoagulant drugs and where possible should provide laboratory services at these clinics to enable dose adjustment to be made on the spot (advising on dose by telephone is to be avoided). The work of these clinics should be confined to the supervision and regulation of anticoagulant dose and related problems. A capillary blood prothrombin time technique is useful for outpatient clinics as it reduces the overall time for testing.

A request to the haematologist for anticoagulant supervision and dose should be in writing and should be signed. An outpatient anticoagulant request card giving patient details and proposed duration of treatment, together with the list of drugs given concomitantly, ideally should be provided with each request.

Patients should be issued with national anticoagulant booklets for the recording of INR results and anticoagulant dose. Supplies of these booklets are available from: DHSS Stores, No 2 Site, Manchester Road, Heywood, Lancashire, OL10 2PZ, or SHHD (Div. IIID), Room 9, St Andrews House, Edinburgh, EH1 3DE. There should be regular correspondence between the clinic doctor and the patient's general practitioner. The practitioner should be informed of non-attendance, any problems associated with treatment, and of a decision to withdraw treatment.

Outpatient anticoagulant clinics should be staffed by adequate numbers of medical, nursing, scientific and secretarial staff. Full records of patients' attendances, results, anticoagulant dosing and correspondence should be kept in special anticoagulant clinic case records.

The dose should also be recorded in the patient's booklet and on the local record. This information should ideally be conveyed to the 
patient while in the anticoagulant clinic but where for logistic reasons this is not possible, the recorded dose in the booklet should be posted to the patient as soon as possible.

\section{QUALITY CONTROL OF ANTICOAGULANT} ADMINISTRATION

This should be carried out either by determining the proportion of patients within their relevant therapeutic range or by determining the mean perentage adequately treated, with the coefficient of variation (CV) for each therapeutic range. More detailed therapeutic quality control has been advocated in which assessment of the effective ratio for control defined as the mid-point between two estimations is determined. ${ }^{18}$

Laboratory control of oral anticoagulants STANDARDISATION OF THE PROTHROMBIN TIME IN ANTICOAGULANT CONTROL

The coagulation defect induced by oral anticoagulant treatment is best quantified by the INR. The INR is the prothrombin ratio, which it is calculated, would have been obtained with the WHO primary international reference preparation.

\section{INTERNATIONAL REFERENCE PREPARATIONS (IRP)}

The primary WHO international reference thromboplastin-human brain $67 / 40$ combined-was replaced by a second WHO IRP of human brain plain, British Comparative Thromboplastin batch 253 (1983). Additional IRP of rabbit brain plain (RBT/79) and bovine combined (OBT/79) were approved by the WHO in the same year.

\section{CALIBRATION OF THROMBOPLASTIN REAGENTS}

An orthogonal regression analysis is the recommended WHO procedure to compare different thromboplastin reagents and derive equivalent INR. A rectilinear relation of the logarithms of the prothrombin times is used. The slope of the correlation indicates the correlation of the local thromboplastin with the IRP. ${ }^{1-31920}$ This is termed the International Sensitivity Index (ISI). IRP results are plotted on the vertical axis against the logarithms of prothrombin times obtained with the thromboplastin to be calibrated on the same set of plasmas (from 20 normal subjects and 60 stable anticoagulated patients). An ISI can be assigned to each batch of thromboplastin. Prothrombin time results should be given as INR, which may be derived from one of the following formulae, when the ISI is known.

INR $=\operatorname{antilog}(\log ($ prothrombin ratio $) \times I S I)$ or

$$
\mathrm{INR}=(\text { prothrombin ratio })^{\mathrm{ISI}}
$$

With some reagents, INR values are reliably obtained from a chart produced by the manufacturer, but with others they are derived from one of the above equations. To determine the normal control value the NEQAS Steering Committee in Blood Coagulation has recommended that the geometric mean of a minimum of 20 normal controls should be used. The normal control values should be obtained from

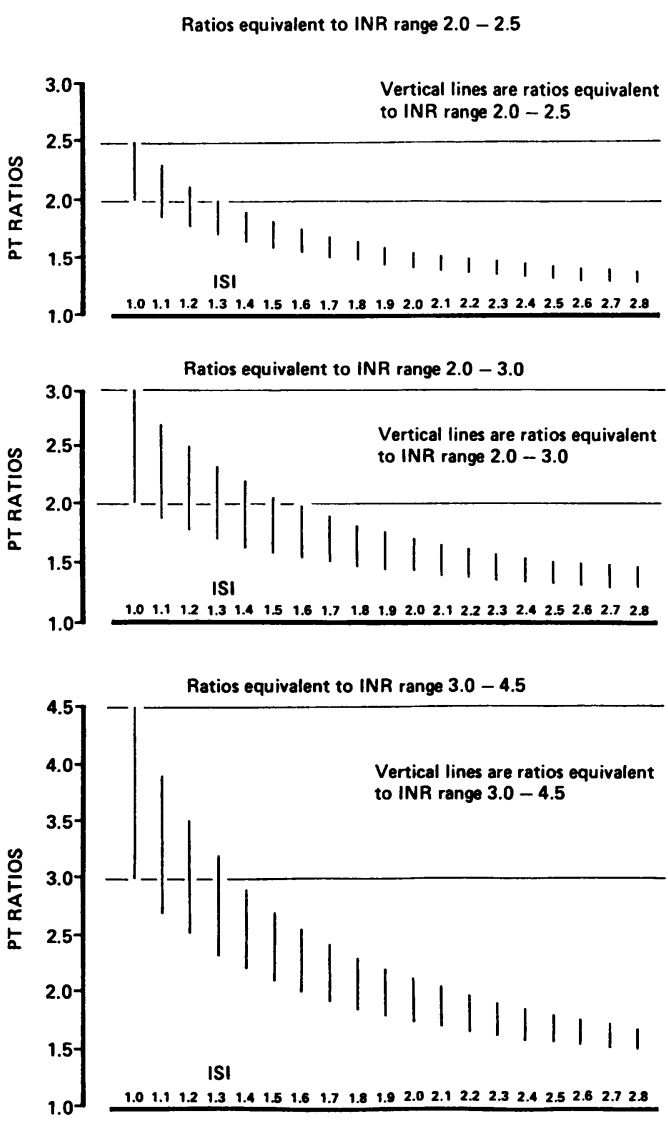

Therapeutic prothrombin ratios equivalent to INR ranges related to increasing ISI of thromboplastins. PT ratios are given for each ISI.

healthy ambulant adults, men and women, and need not necessarily be tested on one day. A locally determined geometric normal mean should be compared with the mean normal value for each batch of thromboplastin issued by the manufacturer, and this may be used to determine the INR. In view of the heavy demands and complexity of the procedure, ISI calibrations should not normally be performed by routine hospital laboratories and should only be performed by National Control Laboratories or by manufacturers. The ISI calibration procedure is described in detail elsewhere. ${ }^{1920}$

\section{THE CLINICAL IMPORTANCE OF A LOW}

ISI THROMBOPLASTIN

The recommended $\mathrm{BSH}$ therapeutic ranges have been based on clinical trials and cumulative experience in the United Kingdom with thromboplastins of ISI between 1.0 and $1 \cdot 1$. NEQAS surveys have shown that these give a lower incidence of persistent poor performance and a lower CV of the INR-that is, increased precision. ${ }^{21}$ Of clinical importance is that the width of the therapeutic range diminishes progressively with increasing ISI (figure).

The INR value may also be less dependable with higher ISI reagents in the induction period of oral anticoagulation and in unstabilised patients.

\section{USE OF COAGULOMETERS}

ISI determinations are based on prothrombin time values obtained with a manual technique. According to 1988 NEQAS data, almost $50^{\circ}$, 
of United Kingdom prothrombin times are performed using a coagulometer. Coagulometers affect the INR value to varying degrees. NEQAS surveys indicate that most types of coagulometer tend to underestimate the INR whereas a minority overestimate the INR. There is, however, considerable variation in performance with instruments of the same model which means that it is not generally safe to apply an ISI correction factor for a type of coagulometer or coagulometer/reagent system as each instrument must be checked individually.

\section{CONTROL BY CHROMOGENIC (AMIDOLYTIC) SUBSTRATES}

Amidolytic assays based on synthetic chromogenic substrates have been recommended for oral anticoagulant dose control. The chromogenic assays are relatively specific to one clotting factor and may measure acarboxy forms. No single chromogenic substrate technique seems to be satisfactory for the control of short-term anticoagulant administration, but specific factor II, VII, and factor $\mathrm{X}$ chromogenic methods give a reasonable approximation to the prothrombin time in long-term stabilised patients. ${ }^{22}$

USE OF COMPUTERS

Computers may play a valuable part in patient records, administration of clinic appointments, and analysis of therapeutic quality control. Their value in production of dose schedules for individual patients is under investigation. Their use for the latter cannot yet be recommended until reliable patient databased programmes have been developed.

\section{Appendix}

DRUGS REPORTED AS INTERACTING WITH ORAL ANTICOAGULANTS

The following preparations should not be regarded as contraindicated or as contraindications to warfarin administration. Their prescription may, however, cause changes in oral anticoagulant requirements. Caution is therefore advised and more frequent monitoring may be required. This list is not intended to be comprehensive. Some drugs have only been referred to in single case reports.

\section{Potentiating drugs \\ Antagonistic drugs \\ Gastrointestinal tract: \\ Antacids-magnesium salts \\ Chloestyramine Colestipol \\ Cimetidine \\ Liquid paraffin and other laxatives}

Cardiovascular system:

Amiodarone

Clofibrate

Dextrothyroxine

Diazoxide

Dipyridamole

Ethacrynic acid

Quinindine

Sulphinpyrazone
Respiratory system:

Central nervous system:

Chloral hydrate and related compounds

Chlorpromazine

Dextropropoxyphene

Dichloralphenazoneinitial

Diflunisal

Mefenamic acid

Monoamine oxidase inhibitors

Tricyclic antidepressants

Triclofos sodium

Infections:

Aminoglycosides:

Griseofulvin

Amikacin

Rifampicin

Gentamicin

Kanamycin

Neomycin

Streptomycin

Co-trimoxazole

Tobramycin

Cephalosporins:

Cephaloridine

Cephazolin

Cephamandole

Chloramphenicol

Latamoxef

Cycloserine

Erythromycin

Isoniazid

Ketoconazole

Metronidazole

Miconazole

Nalidixic acid

Penicillin G-large doses-intravenous

Ampicillin-oral

Quinine salts

Streptotriad

Sulphonamides-long acting

Tetracycline

Endocrine system:

Anabolic steroids

Chlorpropamide

Oral contraceptives

Corticosteroids

Danazol

Glucagon

Metoclopramide

Propylthiouracil

Sulphonyl urea

Thyroxine

Tolbutamide

Malignant disease and immunosuppression:

Cyclophosphamide

Mercaptopurine

Methotrexate

Immunosuppressant drugs

Tamoxifen

Musculoskeletal and joint disease:

Allopurinol

Aspirin and the salicylates

Azapropazone

Diflunisal

Fenclofenac

Fenoprofen

Feprazone 
Flufenamic acid

Flurbiprofen

Indomethacin

Ketoprofen

Mefenamic acid

Naproxen

Paracetamol-high daily doses (with dextropropoxyphene Distalgesic/coproxamol)

Piroxicam

Sulindac

Sulphinpyrazone

Nutrition and blood:

Alcohol-dose dependent potentiator

Ear, nose, and oesophagus:

Skin:

Alcoholism:
Vitamin K

Alcohol

\section{Antihistamines}

Phenazone

Antihistamines
Disulfiram (Antabuse)

1 Lewis SM. Thromboplastin and oral anticoagulant control. Br J Haematol 1987;66:1-4.

2 Shinton NK. Standardisation of oral anticoagulant therapy. Br Med J 1983;287:1000-1.

3 Loeliger EA, Poller L, Samama M, et al. Questions and answers on prothrombin time standardisation in oral anticoagulant control. Thromb Haemostas 1985;54: 515-17.

4 Sevitt $S$. Venous thrombosis and pulmonary embolism. Their prevention by oral anticoagulation. $A m \mathrm{~J} \mathrm{Med}$ 1962;33:703.

5 Taberner DA, Poller L, Burslem RW, Jones JB. Oral anticoagulants controlled by the British Comparative
Thromboplastin versus low-dose heparin prophylaxis of deep vein thrombosis. Br Med J 1978;11:272.

6 Francis CW, Marder VI, McCollister EC, et al. Two step warfarin therapy. JAMA 1983;249:374-8.

7 Resenekow L, Chediak J, Hirsh J, Lewis HD. Antithrombotic agents in coronary heart disease. Chest 1989;95: $52 S-72 S$.

8 Hyers TS, Hull RD, Weg JC. Antithrombotic therapy for venous thromboembolic disease. Chest 1989;95:37S-51S. 9 Stein PD, Kantrowitz A. Antithrombotic therapy in saphenous vein by-pass grafts. Chest 1989;95:107S-117S

10 Gohlke H, Gohlke-Barwolf C, Sturzenhofecker P, et al. Improved graft patency with anticoagulant therapy after coronary artery bypass surgery: a prospective randomised study. Circulation 1981;64(Suppl II):22-7.

11 Petersen P, Boysen G, Godtfredsen J, et al. Placebo controlled randomised trial of warfarin and aspirin for prevention of thromboembolic complications in chronic prevention of thromboembolic complicat

12 Hirsh J, Poller L, Deykin D, et al. Optimal therapeutic range for oral anticoagulants. Chest 1989;95:5S-11S.

13 Hull R, Hirsh J, Jay R, et al. Different intensities of oral anticoagulant therapy in the treatment of proximal-vein thrombosis. N Engl J Med 1982;307:1676.

14 Medical Research Council. Assessment of short term anticoagulant administration after cardiac infarction. $B r$ Med J 1969;1:1335-42.

15 Veterans Administration. Anticoagulants in acute myocardial infarction. $J A M A$ 1973;222:724-9.

16 Sixty-Plus Reinfarction Study Group. A double blind trial to assess long-term oral anticoagulant therapy in elderly
patients after myocardial infarction. Lancet 1980;ii: patients

17 Turpie CG, Gunstensen J, Hirsh J, Nelson H, Gent M. Randomised comparison of two intensities of ora anticoagulant therapy after tissue heart valve replacement. Lancet 1988; ;:124-5.

18 Duxbury BMcD. Therapeutic control of anticoagulant treatment. Br Med J 1982;284:702.

19 Kirkwood TBL. Calibration of reference thromboplastins and standardisation of the prothrombin ratio. Thromb Haemostas 1983;49:238-44.

20 Tomensen J, Thomson JM. Standardisation of the prothrombin time in blood coagulation and haemostasis. In rombin time in blood coagulation and haemostasis. In: Thomson JM, ed. Blood Coagulation and Haemostasis. 3

21 Poller L, Taberner DA, Thomson JM, Darby KV. Survey of prothrombin time results in National External Quality Assessment Scheme exercises 1980-87. J Clin Pathol 1988;41:361-4.

22 O'Donnell JR, Walker ID, Davidson JF. Control of oral anticoagulant therapy with a chromogenic prothrombin assay. Br J Haematol 1983;55:172-5. 
Lecture Notes on Forensic Medicine. 5th ed. DJ Gee, AA Watson. (Pp 229; paperback £12.95.) Blackwells. 1989. ISBN 0-63202595-6.

This paperback makes a very welcome return in its fifth edition and is mostly unscathed with only some loss of width, a gain in height, and the acquisition of a blushing Aesculapian serpent on its front cover: there is a decrease in the number of its chapters (30), pages (220) and in the breadth of the subjects covered. In this edition, regius Professor Alan Watson has joined emeritus Professor David Gee as a coauthor adding a modicum of Scottish law at the appropriate places and further enhancing the book's appeal to the countries on both sides of Hadrian's Wall. Presumably the reason why such subjects as criminal abortion are still covered at length also stems from the necessity to retain a transcontinental clientele.

The "Lecture Notes" series is specifically intended for undergraduates and recently fledged doctors and there can be no doubt that this role is fulfilled most admirably in the specialty of forensic medicine through the commendable conciseness and clarity of text, the subdivision of the chapters by numerous subheadings, and the informative inclusion of simple illustrative line drawings-all at a price (slightly inflated from the last edition) easily accessible to a student's pocket.

The authors seem to have placed great faith on their previous reviewers and I therefore tentatively venture to suggest some changes in emphasis for the forthcoming edition: breath alcohol-assaying machines are here to stay-and child non-accidental injury and sexual abuse will persist in the limelight. The nuances of (Scottish) precognitions and fatal accident inquiries could be highlighted even further. I also hope that the pious hope expressed that the Procurator Fiscal "will always require an autopsy to be carried out" comes to pass by then.

A BUSUTTIL

\section{ERRATA}

\section{Errata 1}

In the indexed letter, "Lack of in vitro activity of omeprazole against Campylobacter pylori," A M Gheleni et al (1990;43:171). The figure legend to the figure reproduced below was inadvertently omitted. We apologise for this error.

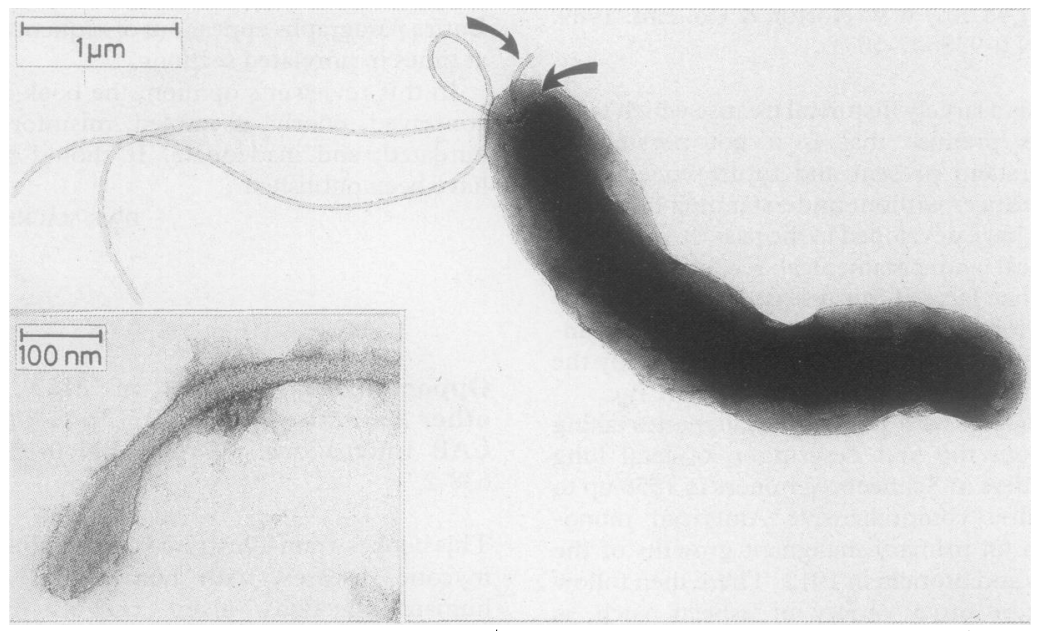

Electron micrograph showing $C$ pylori negatively stained with ammonium molybdate. Although only two intact flagella are seen here, the basal portions of two other flagella are visible (arrowed). A terminal paddle is just visible on one of the two intact flagella. A terminal paddle from another specimen is shown enlarged in the inset.

\section{Errata 2}

Part of the Appendix in "Guidelines on oral anticoagulation: second edition" by the British Society for Haematology $(1990 ; 43: 177-84)$ was incorrectly transcribed. The correct version is printed below. We apologise for this error.

\section{Infections: \\ Aminoglycosides: \\ Amikacin \\ Gentamicin \\ Kanamycin \\ Neomycin \\ Streptomycin \\ Tobramycin \\ Co-trimoxazole \\ Cephalosporins: \\ Cephaloridine \\ Cephazolin \\ Cephamandole \\ Latamoxef}

Griseofulvin

Rifampicin

Association of Clinical Pathologists

Model Training Programmes

Histopathology/Cytopathology/Morbid Anatomy

Medical Microbiology

Haematology and Blood Transfusion

Chemical Pathology Immunology

The Second revised version of these training programmes has now been published in a single booklet. It offers detailed guidance on training in each of the laboratory medical disciplines with recommended reading lists and learning objectives set for those aspiring to consultant status. The booklet should also be of interest to medical graduates who wish to find out what a career in each of these disciplines entails.

Copies are available from the General Secretary, Association of Clinical Pathologists, School of Biological Sciences,

Falmer, Brighton BN1 9QG, UK.

Price $£ 7.50$ (inclusive of postage and packaging). Cheques/sterling drafts should be made payable to the Association of Clinical Pathologists.

\section{ACP Locum Bureau}

The Association of Clinical Pathologists runs a locum bureau for consultant pathologists.

Applicants with the MRC Path who would like to do locums and anyone requiring a locum should contact $\mathrm{Dr}$ David Melcher, Histopathology Department, Sussex County Hospital, Eastern Road, Brighton BN2 5BE. 